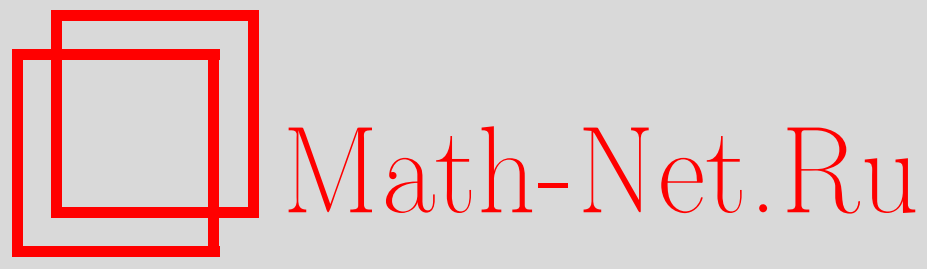

В. Е. Бенинг, В. Ю. Королев, Непараметрическое оценивание вероятности разорения для обобщенных процессов риска, Теория вероятн. и ее примен., 2002, том 47, выпуск 1, 3-20

DOI: https://doi.org/10.4213/tvp2954

Использование Общероссийского математического портала MathNet.Ru подразумевает, что вы прочитали и согласны с пользовательским соглашением

http://www . mathnet.ru/rus/agreement

Параметры загрузки:

IP : 18.209 .158 .208

26 апреля 2023 г., 13:20:00

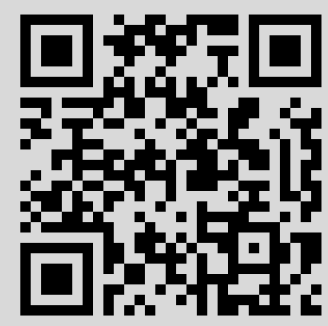


(C) 2002 г.

БЕНИНГ В. Е.*, КОРОЛЕВ В. Ю.*

\section{НЕПАРАМЕТРИЧЕСКОЕ ОЦЕНИВАНИЕ ВЕРОЯТНОСТИ РАЗОРЕНИЯ ДЛЯ ОБОБЩЕННЫХ ПРОЦЕССОВ РИСКА ${ }^{1)}$}

В данной заметке мы строим статистическую оценку вероятности разорения для обобщенного процесса риска, характеризующегося стохастичностью процессов страховых премий и интенсивности страховых выплат. Изучены асимптотические свойства предложенной оценки. Предложены алгоритмы построения приближенных непараметрических доверительных интервалов для вероятности разорения.

Ключевые слова и фразы: процесс риска, обобщенный процесс Кокса, вероятность разорения, случайные последовательности со случайными индексами, несмешенность, состоятельность, доверительные интервалы.

1. Введение. В данной статье, продолжая исследования асимптотических свойств обобщенных процессов риска, начатые в работе [1], мы рассматриваем задачу о статистическом оценивании вероятности разорения для таких процессов и исследуем асимптотические свойства предлагаемых оценок.

Базой для дальнейших обобщений будет служить классический прощесс риска

$$
R_{0}(t)=c t-\sum_{j=1}^{N_{1}(t)} X_{j}, \quad t \geqslant 0,
$$

где $c>0$ - ставка страховой премии, $\left\{X_{j}\right\}_{j \geqslant 1}-$ независимые одинаково распределенные неотрицательные случайные величины с $\mathbf{E} X_{j}=a$, имеюшие смысл страховых выплат, $N_{1}(t)$ - стандартный пуассоновский процесс (однородный пуассоновский процесс с единичной интенсивностью), независимый от $\left\{X_{j}\right\}_{j \geqslant 1}$ и имеющий смысл количества страховых случаев до момента времени $t$. (Мы рассматриваем классический

\footnotetext{
* Московский государственный университет им. М. В. Ломоносова, факультет вычислительной математики и кибернетики, Воробьевы горы, 119899 Москва, Россия; e-mail: vkorolev@df.ru, bening@cs.msu.su

1) Работа выполнена при поддержке Российского фонда фундаментальных исследований (проект 00-01-00360), Российского гуманитарного научного фонда (проект 00-02-00152a), а также Committee on Knowledge Extension Research (CKER) Американского общества актуариев.
} 
процесс риска в форме, немного отличающейся от традиционной. Как правило, предполагается, что процесс возникновения страховых требований - однородный пуассоновский с некоторой интенсивностью $\lambda>0$. Мы же считаем, что $\lambda=1$. Это предположение отнюдь не ограничивает общность наших рассуждений, а означает лишь, что мы выбираем единицу времени так, чтобы в среднем на единицу времени приходилась ровно одна страховая выплата. При этом $c$ имеет смысл прироста капитала страховой компании за выбранную таким образом единицу времени.)

Пусть $\Lambda(t), t \geqslant 0$, - независимый от $N_{1}(t)$ случайный процесс с неубывающими, почти наверное конечными непрерывными справа траекториями, выходящими из нуля. Процесс Кокса (также называемый дважды стохастическим пуассоновским процессом), управляемый процессом $\Lambda(t)$, определяется как

$$
N(t)=N_{1}(\Lambda(t)), \quad t \geqslant 0 .
$$

Рассмотрим процесс

$$
R(t)=c \Lambda(t)-\sum_{j=1}^{N(t)} X_{j}, \quad t \geqslant 0,
$$

в предположении, что процесс $\Lambda(t)$ независим от последовательности $\left\{X_{j}\right\}_{j \geqslant 1}$. Процесс (3) является естественным обобшением классического процесса риска (1) и более гибкой моделью, поскольку модель (3) учитывает как флуктуации риска, так и флуктуации объема портфеля. Можно показать, что при флуктуациях риска (непостоянной интенсивности страховых выплат) в разумных стратегиях страховщика интенсивность пополнения резерва не должна быть постоянной [2], [3]. С другой стороны, очевидно, что среднее число выплат должно быть пропорционально количеству страховых договоров в портфеле страховой компании, которому, в свою очередь, в определенном смысле пропорционально значение $\Lambda(t)$. Следуя [1], процесс $R(t)$ мы будем называть обобщенным процессом риска.

Пусть $s>0$ - начальный капитал страховой компании. Несложно видеть, что вероятность разорения для обобщенного процесса риска

$$
\psi(s)=\mathbf{P}\left\{s+\inf _{t>0} R(t)<0\right\}
$$

совпадает с вероятностью разорения для классического процесса риска

$$
\psi_{0}(s)=\mathbf{P}\left\{s+\inf _{t>0} R_{0}(t)<0\right\},
$$

поскольку процесс $R(t)$ отличается от $R_{0}(t)$ лишь случайной (вообще говоря, неоднородной) компрессией времени, не изменяющей амплитуду траекторий. Мы будем существенно использовать это обстоятельство.

Ниже мы рассмотрим алгоритм построения статистических оценок вероятности разорения и исследуем асимптотические свойства предложенных оценок. 
Символы $\Rightarrow, \stackrel{\mathbf{P}}{\rightarrow}$ и $\stackrel{\mathrm{d}}{=}$ будут обозначать соответственно слабую сходимость, сходимость по вероятности и совпадение распределений. Стандартная нормальная функция распределения будет обозначаться $\Phi(x)$.

2. Статистические оценки вероятности разорения. Известны многие аналитические методы вычисления границ (нижних и верхних оценок) для вероятности разорения. Все они существенно используют информацию о поведении хвостов распределений страховых требований и существенно различны в зависимости от характера убывания этих хвостов. Однако, на практике получить исчерпывающую информацию о поведении хвостов требований, вообе говоря, не представляется возможным, поскольку статистические выводы о распределении страховых требований приходится делать на основе выборки конечного объема, результатом чего является чрезвычайно малая надежность выводов о поведении хвостов распределений выплат для значений аргументов, превосходящих наибольшее наблюдение. Тем самым, с практической точки зрения оказывается очень важной идея построения статистических оценок вероятности разорения (в том числе и верхних и нижних ее доверительных границ) напрямую, без предварительного оценивания хвостов распределений страховых выплат.

Задача статистического оценивания вероятности разорения для обобщенного процесса риска (равно как и для классического процесса риска) по его предыстории до некоторого фиксированного момента $t$ имеет одну важную особенность. А именно, число $N(t)$ страховых выплат, осушествленных до этого момента, случайно. Поэтому на практике вероятность разорения приходится оценивать по выборке $X_{1}, X_{2}, \ldots, X_{N(t)}$ случайного объема. При этом класс возможных распределений случайной величины $N(t)$ даже при описанных выше ограничениях весьма широк и отнюдь не ограничивается только пуассоновскими законами. Например, если $\Lambda(t)$ имеет гамма-распределение, то распределение $N(t)$ является отрицательным биномиальным.

Важным шагом в направлении построения статистических оценок вероятности разорения стала работа Веравербеке и Кру [8], в которой предложена непараметрическая оценка вероятности разорения для классического процесса риска и доказана ее асимптотическая нормальность. Однако элегантные результаты этой работы едва ли могут найти широкое практическое применение, поскольку, во-первых, оценки в ней строятся на основе выборки неслучайного объема и, во-вторых, свойство асимптотической нормальности построенной в работе [8] оценки вероятности разорения нельзя напрямую использовать для построения (асимптотических) доверительных интервалов, поскольку предельное распределение оценки (точнее, его дисперсия) зависит от неизвестного распределения требований. 
Нашей целью является построение практически применимых точечных и интервальных оценок вероятности разорения для обобщенных процессов риска.

Исходя из принципа неразорения в среднем, предположим, что $c>a$. Как мы убедились выше,

$$
\psi(s)=\psi_{0}(s)
$$

Поэтому мы можем использовать хорошо известное представление вероятности разорения для классического процесса риска

$$
\psi_{0}(s)=\left(1-\frac{a}{c}\right) \sum_{k=1}^{\infty}\left(\frac{a}{c}\right)^{k}\left(1-G^{* k}(s)\right),
$$

где $G(x)=a^{-1} \int_{0}^{x}(1-F(y)) d y, x \geqslant 0$, и $G(x)=0$ при $x<0$, $F(y)=\mathbf{P}\left\{X_{1}<y\right\}$, а символ $G^{* k}$ обозначает $k$-кратную свертку функции распределения $G$ с самой собой, $G^{* k}(x)=\left(G^{*(k-1)} * G\right)(x), k \geqslant 1, G^{* 0}-$ функция распределения с единственным единичным скачком в нуле. Соотношение (4) называют формулой Поллачека-Хинчина или формулой Бекмана (см., например, [4], [5], [8]).

Мы будем считать, что параметры $c$ и $a$ известны. Вначале формально предположим, что в нашем распоряжении имеется выборка $X_{1}, \ldots, X_{n}$, где $n \geqslant 1$ - некоторое целое число. Для такой ситуации в работе [8] для $\psi_{0}(s)$ предложена следующая непараметрическая оценка. Поскольку

$$
\psi_{0}(s)=\frac{a}{c}-\left(1-\frac{a}{c}\right) \bar{\psi}_{0}(s)
$$

где

$$
\bar{\psi}_{0}(s)=\sum_{k=1}^{\infty}\left(\frac{a}{c}\right)^{k} G^{* k}(s),
$$

достаточно построить оценку для $\bar{\psi}_{0}(s)$.

Пусть $A \subseteq \mathbb{R}^{1}$. Символом $\mathbf{1}_{A}(x)$ мы будем обозначать индикаторную функцию множества $A: \mathbf{1}_{A}(x)=1$, если $x \in A$, и $\mathbf{1}_{A}(x)=0$, если $x \notin A$. Пусть $Y_{1}, Y_{2}, \ldots$ - независимые одинаково распределенные случайные величины с функцией распределения $G(x)$. Тогда

$$
\begin{aligned}
G^{* k}(s) & =\mathbf{P}\left\{Y_{1}+\cdots+Y_{k}<s\right\} \\
& =\frac{1}{a^{k}} \int_{0}^{\infty} \cdots \int_{0}^{\infty} \mathbf{1}_{[0, s)}\left(y_{1}+\cdots+y_{k}\right) \prod_{j=1}^{k}\left(1-F\left(y_{j}\right)\right) d y_{1} \cdots d y_{k} .
\end{aligned}
$$

Пусть $F_{n}(x)$ - эмпирическая функция распределения, построенная по выборке $X_{1}, \ldots, X_{n}$, т.е.

$$
F_{n}(x)=\frac{1}{n} \sum_{i=1}^{n} \mathbf{1}_{[0, x)}\left(X_{i}\right), \quad x \geqslant 0 .
$$


Тогда, заменяя в соотношении (7) $F$ на $F_{n}$, получим выражение

$$
\frac{1}{n^{k} a^{k}} \sum_{i_{1}=1}^{n} \cdots \sum_{i_{k}=1}^{n} \int_{0}^{\infty} \cdots \int_{0}^{\infty} \mathbf{1}_{[0, s)}\left(y_{1}+\cdots+y_{k}\right) \prod_{j=1}^{k} \mathbf{1}_{\left[y_{j}, \infty\right)}\left(X_{i_{j}}\right) d y_{1} \cdots d y_{k}
$$

которое представляет собой функционал Мизеса (см. [10, с. 32]). Поэтому в качестве статистической оценки для $G^{* k}(s)$ можно рассмотреть $U$-статистику вида

$$
U_{n, k}=U_{n, k}(s)=\left(C_{n}^{k}\right)^{-1} \sum_{1 \leqslant i_{1}<\cdots<i_{k} \leqslant n} h_{k}\left(X_{i_{1}}, \ldots, X_{i_{k}}\right)
$$

с симметричным ядром

$$
\begin{aligned}
& h_{k}\left(x_{1} \ldots, x_{k}\right) \\
& \quad=\frac{1}{a^{k}} \int_{0}^{\infty} \cdots \int_{0}^{\infty} \mathbf{1}_{[0, s)}\left(y_{1}+\cdots+y_{k}\right) \prod_{j=1}^{k} \mathbf{1}_{\left[y_{j}, \infty\right)}\left(x_{j}\right) d y_{1} \cdots d y_{k} .
\end{aligned}
$$

Пусть $m(n)$ - некоторое целое число, $m(n) \leqslant n$. В силу (5) и (6) в качестве оценки для $\psi_{0}(s)$ при неслучайном объеме выборки $n$ формально примем

$$
\psi_{n}(s)=\frac{a}{c}-\left(1-\frac{a}{c}\right) \bar{\psi}_{n}(s)
$$

где

$$
\bar{\psi}_{n}(s)=\sum_{k=1}^{m(n)}\left(\frac{a}{c}\right)^{k} U_{n, k} .
$$

Теперь ясно, что, имея выборку $X_{1}, \ldots, X_{N(t)}$, в качестве оценки для $\psi(s)=\psi_{0}(s)$ следует взять

$$
\psi_{N(t)}(s)=\frac{a}{c}-\left(1-\frac{a}{c}\right) \bar{\psi}_{N(t)}(s)
$$

где

$$
\bar{\psi}_{N(t)}(s)=\sum_{k=1}^{m(N(t))}\left(\frac{a}{c}\right)^{k} U_{N(t), k}
$$

3. Асимптотические свойства оценки вероятности разорения для обобщенного процесса риска. Обозначим

$$
\sigma^{2}=\left(1-\frac{a}{c}\right)^{2} \sum_{k=1}^{\infty} \sum_{m=1}^{\infty}\left(\frac{a}{c}\right)^{k+m} k m \cdot \sigma_{k, m},
$$

где

$$
\begin{aligned}
\sigma_{k, m} & =\mathbf{E} h_{k}\left(X_{1}\right) h_{m}\left(X_{1}\right)-G^{* k}(s) G^{* m}(s), \\
h_{j}(x) & =\frac{1}{a} \int_{0}^{\infty} G^{*(j-1)}(s-y) \mathbf{1}_{[y, \infty)}(x) d y .
\end{aligned}
$$

Следующее утверждение играет главную роль в данном пункте. 
Теорема 1. Пусть оченка $\psi_{N(t)}(s)$ определяется соотношениями (12) и (13), причем Функиия $m(n)$ такова, что $m(n) \rightarrow \infty$ при $n \rightarrow \infty$ mak, чmo

$$
\lim _{n \rightarrow \infty} \frac{\ln n}{m(n)}=0 .
$$

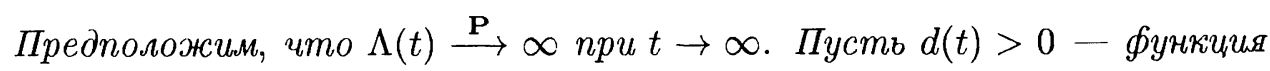
maкал, что $d(t) \rightarrow \infty(t \rightarrow \infty)$. Для того чтобы случайная величина $\sigma^{-1} \sqrt{d(t)}\left(\psi_{N(t)}(s)-\psi(s)\right)$ имела предельное распределение при $t \rightarrow \infty$ :

$$
\sigma^{-1} \sqrt{d(t)}\left(\psi_{N(t)}(s)-\psi(s)\right) \Longrightarrow Z \quad(t \rightarrow \infty)
$$

необходимо и достаточно, чтобы существовала такая неотрицательная случайная величина $Y$, что

$$
\frac{\Lambda(t)}{d(t)} \Longrightarrow Y \quad(t \rightarrow \infty) \text {. }
$$

При этом

$$
\mathbf{P}\{Z<x\}=\mathbf{E} \Phi(x \sqrt{Y}), \quad x \in \mathbb{R}^{1} .
$$

Предельное распределение (20) одно и то же для любого $s>0$.

Доказательству теоремы 1 мы предпошлем несколько лемм.

Лемма 1. Пусть $N(t)$ - прочесс Кокса, управляемый прочес$\operatorname{coм} \Lambda(t)$. Тогда $N(t) \stackrel{\mathbf{P}}{\longrightarrow} \infty$, если и только если $\Lambda(t) \stackrel{\mathbf{P}}{\longrightarrow} \infty$.

Д о к а 3 а т ел ь с т в о см. в [6].

Лемма 2. Пусть $N(t)$ - прочесс Кокса, управляемый прочессом $\Lambda(t)$. Пусть $d(t)>0-\oint$ ункиия такая, ито $d(t) \rightarrow \infty(t \rightarrow \infty)$. Тогда для того итобы одномерные распределения нормированного проиесса Кокса слабо сходились к распределению некоторой случайной величины $Y$ :

$$
\frac{N(t)}{d(t)} \Longrightarrow Y \quad(t \rightarrow \infty)
$$

необходимо и достаточно, чтобы одномерные распределения нормированного управляющего прочесса $\Lambda(t)$ слабо сходились $к$ тому же распределению:

$$
\frac{\Lambda(t)}{d(t)} \Longrightarrow Y \quad(t \rightarrow \infty)
$$

Д ок а з а т е л ь с т в о см. в [6] и [7].

Лемма 3. Пусть оченка $\psi_{n}(s)$ определяется соотношениями (10) $u(11)$, причем Функиия $m(n) \leqslant n$ неограниченно возрастает $n p u n \rightarrow \infty$ так, что выполнено (17). Тогда случайная величина $\sigma^{-1} \sqrt{n}\left(\psi_{n}(s)\right.$ $\left.\psi_{0}(s)\right)$ асимптотически нормальна:

$$
\mathbf{P}\left\{\sigma^{-1} \sqrt{n}\left(\psi_{n}(s)-\psi_{0}(s)\right)<x\right\} \Longrightarrow \Phi(x) \quad(n \rightarrow \infty) .
$$


Д ок а з а т е л с с т в ом. в [8].

Рассмотрим случайные величины $N_{1}, N_{2}, \ldots, X_{1}, X_{2}, \ldots$, определенные на одном и том же измеримом пространстве $(\Omega, \mathscr{A})$. Пусть на $\mathscr{A}$ задано семейство вероятностных мер $\left\{\mathbf{P}_{\theta}, \theta \in \Theta\right\}$. Предположим, что при каждом $n \geqslant 1$ случайная величина $N_{n}$ принимает только натуральные значения и независима от последовательности $X_{1}, X_{2}, \ldots$ относительно каждой меры из семейства $\left\{\mathbf{P}_{\theta}, \theta \in \Theta\right\}$. Пусть $T_{n}=T_{n}\left(X_{1}, \ldots, X_{n}\right)-$ некоторая статистика. Для каждого $n \geqslant 1$ определим случайную величину $T_{N_{n}}$, положив $T_{N_{n}(\omega)}=T_{N_{n}(\omega)}\left(X_{1}(\omega), \ldots, X_{N_{n}(\omega)}(\omega)\right)$ для каждого элементарного исхода $\omega \in \Omega$. Будем говорить, что статистика $T_{n}$ асимптотически нормальна, если существуют функции $\delta(\theta)$ и $t(\theta)$ такие, что при каждом $\theta \in \Theta$

$$
\mathbf{P}_{\theta}\left\{\delta(\theta) \sqrt{n}\left(T_{n}-t(\theta)\right)<x\right\} \Longrightarrow \Phi(x) \quad(n \rightarrow \infty) .
$$

Лемма 4. Пусть $\left\{d_{n}\right\}_{n \geqslant 1}$ - некоторая неограниченно возрастаюшая последовательность положительных чисел. Предположим, что $N_{n} \stackrel{\mathbf{P}}{\rightarrow} \infty$ nрu $n \rightarrow \infty$. Пусть статистика $T_{n}$ асимптотически нормальна в смысле (21). Для того чтобы при каждом $\theta \in \Theta$ существовала такая функиия распределения $F(x, \theta)$, ито

$$
\mathbf{P}_{\theta}\left\{\delta(\theta) \sqrt{d_{n}}\left(T_{N_{n}}-t(\theta)\right)<x\right\} \Longrightarrow F(x, \theta) \quad(n \rightarrow \infty),
$$

необходимо и достаточно, чтобы существовало семейство функиий распределения $\mathscr{H}=\{H(x, \theta): \theta \in \Theta\}$, удовлетворяюшее условиям

$$
\begin{gathered}
H(x, \theta)=0, \quad x<0, \theta \in \Theta ; \\
F(x, \theta)=\int_{0}^{\infty} \Phi(x \sqrt{y}) d_{y} H(y, \theta), \quad x \in \mathbb{R}^{1}, \theta \in \Theta ; \\
\mathbf{P}_{\theta}\left\{N_{n}<d_{n} x\right\} \Longrightarrow H(x, \theta), \quad n \rightarrow \infty, \theta \in \Theta .
\end{gathered}
$$

При этом если функиии распределения случайньх величин $N_{n}$ не зависят от $\theta$, то не зависят от $\theta$ и функиии распределения $H(x, \theta)$, т.е. семейство $\mathscr{H}$ состоит из единственного элемента.

Д о к а з а т е л ь с т в о. Данная лемма по сути лишь переобозначениями отличается от теоремы 3 из [9].

Д ок а з а т ел ь с т в о т е о р е мы 1. Пусть $\left\{t_{1}, t_{2}, \ldots\right\}-$ произвольная неограниченно возрастающая последовательность моментов времени. Положим $N_{n}=N\left(t_{n}\right), n \geqslant 1$. По лемме 1 условия $\Lambda(t) \stackrel{\mathbf{P}}{\longrightarrow} \infty$ и $N(t) \stackrel{\mathbf{P}}{\longrightarrow} \infty$ эквивалентны при $t \rightarrow \infty$. Поэтому, так как, согласно лемме 3 , оценка $\psi_{n}(s)$ асимптотически нормальна, то по лемме 4 для сходимости (18), в которой $t$ пробегает последовательность $\left\{t_{1}, t_{2}, \ldots\right\}$, необходимо и достаточно, чтобы сушествовала случайная величина $Y \geqslant 0$ такая, что

$$
\frac{N_{n}}{d\left(t_{n}\right)} \Longrightarrow Y \quad(n \rightarrow \infty)
$$


Но по лемме 2 сходимость (22) имеет место тогда и только тогда, когда

$$
\frac{\Lambda\left(t_{n}\right)}{d\left(t_{n}\right)} \Longrightarrow Y \quad(n \rightarrow \infty) \text {. }
$$

Как известно, семейство масштабных смесей нормальных законов (20) идентифицируемо, т.е. из того, что $\mathbf{E} \Phi\left(W_{1} x\right)=\mathbf{E} \Phi\left(W_{2} x\right), x \in \mathbb{R}^{1}$, для неотрицательных случайных величин $W_{1}$ и $W_{2}$, вытекает, что $W_{1} \stackrel{\mathrm{d}}{=} W_{2}$ (см., например, [6]). Поэтому распределение случайной величины $Y$ не зависит от выбора последовательности $\left\{t_{1}, t_{2}, \ldots\right\}$. Из произвольности последовательности $\left\{t_{n}\right\}_{n \geqslant 1}$ вытекает, что (23) эквивалентно (19). Теорема доказана.

Следствие 1. В условиях теоремы 1 для асимптотической нормальности оченки $\psi_{N(t)}(s)$ nрu $t \rightarrow \infty$ :

$$
\mathbf{P}\left\{\sigma^{-1} \sqrt{d(t)}\left(\psi_{N(t)}(s)-\psi(s)\right)<x\right\} \Longrightarrow \Phi(x) \quad(t \rightarrow \infty)
$$

необходимо и достаточно, чтобы

$$
\frac{\Lambda(t)}{d(t)} \stackrel{\mathbf{P}}{\longrightarrow} 1 \quad(t \rightarrow \infty) .
$$

Из теоремы 1 мы можем сделать несколько выводов об условиях состоятельности и асимптотической несмешенности оценки $\psi_{N(t)}(s)$. A именно, справедливы следующие утверждения.

Следствие 2. Пусть выполнены условия теоремы 1 и существуют неограниченно возрастающая Функчия $d(t)$ и случайная величина $Y$ такие, что имеет место сходимость (19). Тогда оиенка $\psi_{N(t)}(s)$ состоятельна.

Д о к а з а т е л ь с т в о. Функцию распределения случайной величины $\sigma^{-1} \sqrt{d(t)}\left(\psi_{N(t)}(s)-\psi(s)\right)$ обозначим $\Psi_{t}(x)$. Для произвольного $\varepsilon>0$ имеем

$$
\begin{aligned}
\mathbf{P} & \left\{\left|\psi_{N(t)}(s)-\psi(s)\right|>\varepsilon\right\} \\
& =\mathbf{P}\left\{\sigma^{-1} \sqrt{d(t)}\left|\psi_{N(t)}(s)-\psi(s)\right|>\varepsilon \sigma^{-1} \sqrt{d(t)}\right\} \\
& =\Psi_{t}\left\{-\varepsilon \sigma^{-1} \sqrt{d(t)}\right\}+1-\Psi_{t}\left\{\varepsilon \sigma^{-1} \sqrt{d(t)}+0\right\} .
\end{aligned}
$$

Но, согласно теореме 1 , в условиях следствия 2 семейство функций распределения $\left\{\Psi_{t}(x)\right\}_{t>0}$ слабо компактно вследствие сходимости (18). Это означает, что для любого $\delta>0$ существует такое $R_{\delta}>0$, что, каким бы ни было $t>0$, для любого $R \geqslant R_{\delta}$ справедливо неравенство $\Psi_{t}(-R)+1-\Psi_{t}(R+0)<\delta$. В частности, это выполнено и для $t \geqslant t_{\varepsilon}=\inf \left\{t: \varepsilon \sigma^{-1} \sqrt{d(t)}>R\right\}$. Таким образом, из (25) следует, что для произвольных $\varepsilon>0$ и $\delta>0$ сушествует $t_{0}=t_{0}(\varepsilon, \delta)$ такое, что $\mathbf{P}\left\{\left|\psi_{N(t)}(s)-\psi(s)\right|>\varepsilon\right\}<\delta$, для всех $t \geqslant t_{0}$, что и означает состоятельность оценки $\psi_{N(t)}(s)$. Следствие доказано. 
Особенностью данной задачи является то, что в случае невырожденной случайной величины $Y$ асимптотическое распределение оценки $\psi_{N(t)}(s)$ имеет более тяжелые хвосты, нежели нормальное распределение.

В качестве примера рассмотрим ситуацию, когда $\Lambda(t)$ имеет показательное распределение с параметром $1 / t$. В этом случае разумно положить $d(t) \equiv t$. Тогда распределение случайной величины $Y$ из (19) будет стандартным показательным, и, следовательно, распределение случайной величины $Z$ из (18) имеет вид

$$
\begin{aligned}
\mathbf{P}\{Z<x\} & =\int_{0}^{\infty} \Phi(\sqrt{y} x) e^{-y} d y=\int_{0}^{\infty}\left[\frac{1}{2}+\frac{1}{\sqrt{2 \pi}} \int_{0}^{\sqrt{y} x} e^{-u^{2} / 2} d u\right] e^{-y} d y \\
& =\frac{1}{2}+\frac{1}{\sqrt{2 \pi}} \int_{0}^{\infty} \int_{0}^{\sqrt{y} x} \exp \left\{-\frac{u^{2}}{2}-y\right\} d u d y \\
& =\frac{1}{2}+\frac{1}{\sqrt{2 \pi}} \int_{0}^{\infty} \int_{u^{2} / x^{2}}^{\infty} e^{-y} d y e^{-u^{2} / 2} d u \\
& =\frac{1}{2}+\frac{1}{\sqrt{2 \pi}} \int_{0}^{\infty} \exp \left\{-\frac{u^{2}}{2}\left(1+\frac{2}{x^{2}}\right)\right\} d u \\
& =\frac{1}{2}\left(1+\frac{x}{\sqrt{2+x^{2}}}\right) .
\end{aligned}
$$

У этого распределения отсутствуют моменты порядка $k \geqslant 2$. Несложно видеть, что для $\frac{1}{2}<\beta<1 \quad \beta$-квантиль этого распределения равна $\sqrt{2}(2 \beta-1) / \sqrt{1-(2 \beta-1)^{2}}$. Поэтому, например, расстояние между квантилями порядков 0.975 и 0.025 этого распределения (что в определенном смысле соответствует длине «наикратчайшего доверительного интервала») оказывается почти в 2.2 раза больше соответствующей характеристики нормального распределения с тем же параметром масштаба. Этот пример наглядно иллюстрирует, насколько важно учитывать случайность объема выборки, по которой оценивается тот или иной параметр, в данном случае - вероятность разорения. В противном случае можно существенно ошибиться в реальной точности оценок или в их реальной надежности (легко видеть, что реальная доверительная вероятность «95-процентного нормального» интервала, вычисленная по распределению (26), оказывается меньшей, чем 0.82).

В то же время, если $N(t)=N_{1}(t)$, т.е. если $\Lambda(t) \equiv t$, что соответствует классическому процессу риска, то, как вытекает из следствия 1 с $d(t) \equiv t$, статистика $\psi_{N(t)}(s)$ является асимптотически нормальной. Другими словами, для такой ситуации оценка вероятности разорения, построенная по выборке $\left(X_{1}, \ldots, X_{N_{1}(n)}\right)$, асимптотически $($ при $n \rightarrow \infty)$ эквивалентна оценке вероятности разорения $\psi_{n}(s)$, определенной соотношениями (10) и (11). 
Из-за упомянутой выше особенности предельных законов (наличие тяжелых хвостов, что может выражаться в отсутствии моментов, в частности, математического ожидания) говорить об асимптотической несмещенности оценки $\psi_{N(t)}(s)$ в терминах моментов не всегда целесообразно. Тем не менее, оказывается справедливым следующее утверждение. (Как обычно, медиана случайной величины $X$ будет обозначаться $\operatorname{med} X$.)

Следствие 3. Пусть выполнены условия теоремы 1 и существуют неограниченно возрастающая функиия $d(t)$ и случайная величина $Y$ такие, что имеет место сходимость (19). Тогда оченка $\psi_{N(t)}(s)$ является асимптотически несмещенной в том смысле, что

$$
\lim _{t \rightarrow \infty} \operatorname{med} \psi_{N(t)}(s)=\psi(s) .
$$

Более того,

$$
\operatorname{med} \psi_{N(t)}(s)=\psi(s)+o\left((d(t))^{-1 / 2}\right)
$$

Д ок а з а те ль с тв о. В условиях следствия 3 , согласно теореме 1, имеет место сходимость (18), из которой, как легко видеть, вытекает, что

$$
\lim _{t \rightarrow \infty} \operatorname{med}\left(\sigma^{-1} \sqrt{d(t)}\left(\psi_{N(t)}(s)-\psi(s)\right)\right)=\operatorname{med} Z=0,
$$

откуда следует, что

$$
\sigma^{-1} \sqrt{d(t)}\left(\operatorname{med} \psi_{N(t)}(s)-\psi(s)\right) \rightarrow 0
$$

что в силу неограниченного возрастания функции $d(t)$ возможно только в случае, когда выполнено (27). Далее, соотношение (29) означает справедливость (28). Следствие доказано.

\section{4. Непараметрическая оценка асимптотической дисперсии.} К сожалению, теоремой 1 нельзя напрямую воспользоваться для построения асимптотических доверительных интервалов, поскольку асимптотическая дисперсия $\sigma^{2}$, определяемая соотношениями (14)-(16), неизвестна, будучи зависящей от неизвестной функции распределения $F(x)$. Имея в качестве конечной цели построение асимптотических доверительных интервалов для вероятности разорения $\psi(s)$, в данном пункте мы сосредоточим внимание на построении непараметрической оценки для $\sigma^{2}$ и изучении ее свойств.

Как и в п. 2, вначале мы чисто формально предположим, что в нашем распоряжении имеется выборка $X_{1}, \ldots, X_{n}$ независимых одинаково распределенных наблюдений. Пусть $\{k(n)\}_{n \geqslant 1}$ - последовательность натуральных чисел, удовлетворяюшая условиям: $1 \leqslant k(n) \leqslant n$, $k(n) \rightarrow \infty$ при $n \rightarrow \infty$ и

$$
\frac{1}{\sqrt{n}} \sum_{r=1}^{k(n)}\left(\frac{s}{c}\right)^{r} r^{3 / 2} \cdot \max \left\{1, \sum_{l=1}^{k(n)}\left(\frac{s}{c}\right)^{l} l\right\} \longrightarrow 0 \quad(n \rightarrow \infty) \text {. }
$$


В качестве ощенки $\sigma^{2}$ рассмотрим выражение

$$
\sigma_{n}^{2}=\left(1-\frac{a}{c}\right)^{2} \sum_{r=1}^{k(n)} \sum_{l=1}^{k(n)}\left(\frac{a}{c}\right)^{r+l} r l \bar{\sigma}_{r, l}
$$

где

$$
\begin{aligned}
\bar{\sigma}_{r, l} & =\frac{1}{n} \sum_{i=1}^{n} \bar{h}_{r}\left(X_{i}\right) \bar{h}_{l}\left(X_{i}\right)-U_{n, r} U_{n, l}, \\
\bar{h}_{j}(x) & =\frac{1}{a} \int_{0}^{\infty} U_{n, j-1}(s-y) \mathbf{1}_{[y, \infty)}(x) d y
\end{aligned}
$$

а $U_{n, k}=U_{n, k}(s)$ и $h_{k}\left(x_{1}, \ldots, x_{k}\right)$ определяются соотношениями (8) и (9).

Лемма 5. Пусть последовательность натуральных чисел $\{k(n)\}_{n \geqslant 1}$ удовлетворяет условиям $1 \leqslant k(n) \leqslant n, k(n) \rightarrow \infty u(30)$. Тогда оченка $\sigma_{n}^{2}$ является состоятельной:

$$
\sigma_{n}^{2} \stackrel{\mathrm{P}}{\rightarrow} \sigma^{2} \quad(n \rightarrow \infty) .
$$

Д ок а з а тель с т в. Заметим, что из определения величин $h_{j}(x)$ и $\sigma_{r, l}($ см. (15) и $(16))$ непосредственно вытекают оценки

$$
0 \leqslant h_{j}(x) \leqslant \frac{s}{a}, \quad\left|\sigma_{r, l}\right| \leqslant\left(\frac{s}{a}\right)^{2}+1
$$

Поэтому из сходимости ряда

$$
\sum_{r=1}^{\infty} \sum_{l=1}^{\infty}\left(\frac{a}{c}\right)^{r+l} r l=\frac{(a / c)^{2}}{(1-a / c)^{4}}
$$

следует сходимость ряда $\sum_{r=1}^{\infty} \sum_{l=1}^{\infty}(a / c)^{r+l} r l \sigma_{r, l}$, и значит,

$$
\bar{\sigma}_{n}^{2}=\left(1-\frac{a}{c}\right)^{2} \sum_{r=1}^{k(n)} \sum_{l=1}^{k(n)}\left(\frac{a}{c}\right)^{r+l} r l \sigma_{r, l} \longrightarrow \sigma^{2} \quad(n \rightarrow \infty) .
$$

Поэтому для доказательства соотношения (34) достаточно убедиться, чTO

$$
\sigma_{n}^{2}-\bar{\sigma}_{n}^{2} \stackrel{\mathbf{P}}{\longrightarrow} 0 \quad(n \rightarrow \infty)
$$

Докажем (36). С учетом соотношений (27)-(33) заметим, что для любого $\varepsilon>0$ справедливы неравенства

$$
\begin{aligned}
\mathbf{P}\left\{\left|\bar{\sigma}_{n}^{2}-\sigma_{n}^{2}\right|>\varepsilon\right\} & \leqslant \mathbf{P}\left\{\left(1-\frac{a}{c}\right)^{2} \sum_{r=1}^{k(n)} \sum_{l=1}^{k(n)}\left(\frac{a}{c}\right)^{r+l} r l\left|\bar{\sigma}_{r, l}-\sigma_{r, l}\right|>\varepsilon\right\} \\
& \leqslant A_{n 1}+A_{n 2}+A_{n 3}
\end{aligned}
$$

где

$$
\begin{aligned}
A_{n 1}=\mathbf{P}\left\{\left(1-\frac{a}{c}\right)^{2} \sum_{r=1}^{k(n)} \sum_{l=1}^{k(n)}\left(\frac{a}{c}\right)^{r+l} r l \mid \frac{1}{n} \sum_{i=1}^{n} h_{r}\left(X_{i}\right) h_{l}\left(X_{i}\right)\right. & \\
& \left.-\mathbf{E} h_{r}\left(X_{1}\right) h_{l}\left(X_{1}\right) \mid>\frac{\varepsilon}{3}\right\}
\end{aligned}
$$




$$
\begin{aligned}
& A_{n 2}=\mathbf{P}\left\{\left(1-\frac{a}{c}\right)^{2} \sum_{r=1}^{k(n)} \sum_{l=1}^{k(n)}\left(\frac{a}{c}\right)^{r+l} \frac{r l}{n} \mid \sum_{i=1}^{n}\left[h_{r}\left(X_{i}\right) h_{l}\left(X_{i}\right)\right.\right. \\
& \left.\left.-\bar{h}_{r}\left(X_{i}\right) \bar{h}_{l}\left(X_{i}\right)\right] \mid>\frac{\varepsilon}{3}\right\} \\
& A_{n 3}=\mathbf{P}\left\{\left(1-\frac{a}{c}\right)^{2} \sum_{r=1}^{k(n)} \sum_{l=1}^{k(n)}\left(\frac{a}{c}\right)^{r+l} r l\left|U_{n, r} U_{n, l}-G^{* r}(s) G^{* l}(s)\right|>\frac{\varepsilon}{3}\right\} .
\end{aligned}
$$

Теперь с учетом (37) нам достаточно убедиться, что

$$
A_{n i} \longrightarrow 0 \quad(n \rightarrow \infty), i=1,2,3 .
$$

Докажем (41) при $i=1$. По неравенствам Чебышева и Ляпунова мы имеем

$$
\begin{aligned}
A_{n 1} \leqslant & \frac{3(1-a / c)^{2}}{\varepsilon} \sum_{r=1}^{k(n)} \sum_{l=1}^{k(n)}\left(\frac{a}{c}\right)^{r+l} r l \mathbf{E} \mid \frac{1}{n} \sum_{i=1}^{n} h_{r}\left(X_{i}\right) h_{l}\left(X_{i}\right) \\
& -\mathbf{E} h_{r}\left(X_{1}\right) h_{l}\left(X_{1}\right) \mid \\
\leqslant & \frac{3(1-a / c)^{2}}{\varepsilon} \sum_{r=1}^{k(n)} \sum_{l=1}^{k(n)}\left(\frac{a}{c}\right)^{r+l} r l\left[\mathbf{D}\left(\frac{1}{n} \sum_{i=1}^{n} h_{r}\left(X_{i}\right) h_{l}\left(X_{i}\right)\right)\right]^{1 / 2} \\
\leqslant & \frac{3(1-a / c)^{2}}{\varepsilon \sqrt{n}} \sum_{r=1}^{k(n)} \sum_{l=1}^{k(n)}\left(\frac{a}{c}\right)^{r+l} r l\left[\mathbf{D}\left(h_{r}\left(X_{1}\right) h_{l}\left(X_{1}\right)\right)\right]^{1 / 2} .
\end{aligned}
$$

Чтобы оценить дисперсию, стоящую под знаком суммы в правой части (42), применим неравенство (35) и получим

$$
\mathbf{D}\left(h_{r}\left(X_{1}\right) h_{l}\left(X_{1}\right)\right) \leqslant \mathbf{E} h_{r}^{2}\left(X_{1}\right) h_{l}^{2}\left(X_{1}\right) \leqslant \frac{s^{4}}{a^{4}} .
$$

Поэтому из (42) вытекает соотношение

$$
A_{n 1} \leqslant \frac{3(1-a / c)^{2} s^{2}}{\varepsilon a^{2} \sqrt{n}} \sum_{r=1}^{k(n)} \sum_{l=1}^{k(n)}\left(\frac{a}{c}\right)^{r+l} r l \leqslant \frac{3 s^{2}}{\varepsilon c^{2}(1-a / c)^{2} \sqrt{n}} \longrightarrow 0
$$

при $n \rightarrow \infty$. Докажем теперь справедливость (41) при $i=2$. Из (39) и соотношений $h_{r}(x) h_{l}(x)-\bar{h}_{r}(x) \bar{h}_{l}(x)=h_{l}(x)\left(h_{r}(x)-\bar{h}_{r}(x)\right)+\bar{h}_{r}(x)\left(h_{l}(x)-\right.$ $\left.\bar{h}_{l}(x)\right), 0 \leqslant h_{j}(x) \leqslant s / a, \bar{h}_{j}(x) \leqslant s^{j} / a^{j}$ нетрудно видеть, что

$$
A_{n 2} \leqslant B_{n 1}+B_{n 2}
$$

где

$$
\begin{aligned}
& B_{n 1}=\mathbf{P}\left\{\left(1-\frac{a}{c}\right)^{2} \sum_{r=1}^{k(n)} \sum_{l=1}^{k(n)}\left(\frac{a}{c}\right)^{r+l} r l \sum_{i=1}^{n}\left|h_{r}\left(X_{i}\right)-\bar{h}_{r}\left(X_{i}\right)\right|>\frac{n a \varepsilon}{6 s}\right\}, \\
& B_{n 2}=\mathbf{P}\left\{\left(1-\frac{a}{c}\right)^{2} \sum_{r=1}^{k(n)} \sum_{l=1}^{k(n)}\left(\frac{a}{c}\right)^{r+l} r l \sum_{i=1}^{n}\left|h_{l}\left(X_{i}\right)-\bar{h}_{l}\left(X_{i}\right)\right| \frac{s^{r}}{a^{r}}>\frac{n \varepsilon}{6}\right\} .
\end{aligned}
$$


Оценивая эти величины с помощью неравенств Чебышева и Ляпунова, с учетом (33) мы получаем

$$
\begin{aligned}
B_{n 1} & \leqslant \frac{6 s}{c \varepsilon} \sum_{r=1}^{k(n)} r\left(\frac{a}{c}\right)^{r} \mathbf{E}\left|h_{r}\left(X_{i}\right)-\bar{h}_{r}\left(X_{i}\right)\right| \\
& \leqslant \frac{6 s}{c a \varepsilon} \sum_{r=1}^{k(n)} r\left(\frac{a}{c}\right)^{r} \int_{0}^{s} \mathbf{E}\left|U_{n, r-1}(s-y)-G^{*(r-1)}(s-y)\right| d y \\
& \leqslant \frac{6 s}{c a \varepsilon} \sum_{r=1}^{k(n)} r\left(\frac{a}{c}\right)^{r} \int_{0}^{s}\left[\mathbf{D} U_{n, r-1}(s-y)\right]^{1 / 2} d y .
\end{aligned}
$$

Дисперсию, стоящую под знаком интеграла в правой части (47), оценим с помощью следующего результата из [10] (см. пемму 1.1.4 на с. 27 там). Пусть ядро $H U$-статистики вида

$$
U_{n}=\frac{1}{C_{n}^{m}} \sum_{1 \leqslant i_{1}<\cdots<i_{m} \leqslant n} H\left(X_{i_{1}}, \ldots, X_{i_{m}}\right)
$$

удовлетворяет условию $\mathbf{E} H^{2}\left(X_{1}, \ldots, X_{m}\right)<\infty$. Тогда справедливо неравенство

$$
\mathbf{D} U_{n} \leqslant \frac{m}{n} \mathbf{D} H\left(X_{1}, \ldots, X_{m}\right) .
$$

С учетом (48) мы имеем

$$
\begin{aligned}
\mathbf{D} U_{n, r-1}(s-y) & \leqslant \frac{r-1}{n} \mathbf{D} h_{r-1}\left(X_{1}, \ldots, X_{r-1}\right) \\
& \leqslant \frac{r-1}{n} \mathbf{E} h_{r-1}^{2}\left(X_{1}, \ldots, X_{r-1}\right) .
\end{aligned}
$$

Из (33) легко следует, что

$$
0 \leqslant h_{k}\left(x_{1}, \ldots, x_{k}\right) \leqslant \frac{s^{k}}{a^{k}}
$$

Поэтому из (49) вытекает оценка

$$
\mathbf{D} U_{n, r-1}(s-y) \leqslant \frac{(r-1) s^{2(r-1)}}{n a^{2(r-1)}} .
$$

С учетом (47) и (51) мы имеем

$$
B_{n 1} \leqslant \frac{6 s}{c \varepsilon \sqrt{n}} \sum_{r=1}^{k(n)}\left(\frac{a}{c}\right)^{r} r \sqrt{r-1}\left(\frac{s}{a}\right)^{r} \leqslant \frac{6 s}{c \varepsilon \sqrt{n}} \sum_{r=1}^{k(n)}\left(\frac{s}{c}\right)^{r} r^{3 / 2} \longrightarrow 0
$$

при $n \rightarrow \infty$ в силу условия (30). Аналогично, используя неравенства типа (47) и (51), получим

$$
\begin{aligned}
B_{n 2} & \leqslant \frac{6(1-a / c)^{2}}{\varepsilon} \sum_{r=1}^{k(n)} \sum_{l=1}^{k(n)}\left(\frac{s}{c}\right)^{r}\left(\frac{a}{c}\right)^{l} r l \mathbf{E}\left|h_{l}\left(X_{1}\right)-\bar{h}_{l}\left(X_{1}\right)\right| \\
& \leqslant \frac{6(1-a / c)^{2}}{\varepsilon \sqrt{n}} \sum_{r=1}^{k(n)} \sum_{l=1}^{k(n)}\left(\frac{s}{c}\right)^{r+l} r l^{3 / 2} \longrightarrow 0
\end{aligned}
$$


при $n \rightarrow \infty$ опять-таки в силу условия (30). Осталось убедиться, что соотношение (41) выполняется при $i=3$. С этой целью заметим, что из $(32),(33),(50)$ и соотношений

$$
\begin{aligned}
U_{n, r} U_{n, l}-G^{* r}(s) G^{* l}(s) & =U_{n, l}\left(U_{n, r}-G^{* r}(s)\right)+G^{* r}(s)\left(U_{n, l}-G^{* l}(s)\right), \\
\mathbf{E} U_{n, k} & =G^{* k}(s), \quad G^{* k}(s) \leqslant 1
\end{aligned}
$$

следует, что для правой части (40) справедлива оценка

$$
A_{n, 3} \leqslant B_{n 3}^{(1)}+B_{n 3}^{(2)}
$$

где

$$
\begin{aligned}
& B_{n 3}^{(1)}=\mathbf{P}\left\{\left(1-\frac{a}{c}\right)^{2} \sum_{r=1}^{k(n)} \sum_{l=1}^{k(n)}\left(\frac{a}{c}\right)^{r+l} r l\left|U_{n, l}-G^{* l}(s)\right|>\frac{\varepsilon}{6}\right\}, \\
& B_{n 3}^{(2)}=\mathbf{P}\left\{\left(1-\frac{a}{c}\right)^{2} \sum_{r=1}^{k(n)} \sum_{l=1}^{k(n)}\left(\frac{a}{c}\right)^{r+l} r l\left|U_{n, l}\right|\left|U_{n, r}-G^{* r}(s)\right|>\frac{\varepsilon}{6}\right\} .
\end{aligned}
$$

Оценим $B_{n 3}^{(1)}$ с помошью неравенств Чебышева и Ляпунова:

$$
\begin{aligned}
B_{n 3}^{(1)} & \leqslant \mathbf{P}\left\{\left(1-\frac{a}{c}\right)^{2} \sum_{r=1}^{\infty}\left(\frac{a}{c}\right)^{r} r \sum_{l=1}^{k(n)}\left(\frac{a}{c}\right)^{l} l\left|U_{n, l}-G^{* l}(s)\right|>\frac{\varepsilon}{6}\right\} \\
& =\mathbf{P}\left\{\frac{a}{c} \sum_{l=1}^{k(n)}\left(\frac{a}{c}\right)^{l} l\left|U_{n, l}-G^{* l}(s)\right|>\frac{\varepsilon}{6}\right\} \\
& \leqslant \frac{6 a}{c \varepsilon} \sum_{l=1}^{k(n)}\left(\frac{a}{c}\right)^{l} l \mathbf{E}\left|U_{n, l}-G^{* l}(s)\right| \leqslant \frac{6 a}{c \varepsilon} \sum_{l=1}^{k(n)}\left(\frac{a}{c}\right)^{l} l\left[\mathbf{D} U_{n, l}\right]^{1 / 2} .
\end{aligned}
$$

Чтобы оценить дисперсии, стоящие под знаком суммы в правой части (55), применим неравенства (48)-(51) и получим

$$
B_{n 3}^{(1)} \leqslant \frac{6 a}{c \varepsilon \sqrt{n}} \sum_{l=1}^{k(n)}\left(\frac{s}{c}\right)^{l} l^{3 / 2} \longrightarrow 0
$$

при $n \rightarrow \infty$ в силу условия (30). Теперь рассмотрим $B_{n 3}^{(2)}$. По аналогии с уже доказанным, из (54) мы получим

$$
\begin{aligned}
B_{n 3}^{(2)} & \leqslant \frac{6(1-a / c)^{2}}{\varepsilon} \sum_{r=1}^{k(n)} \sum_{l=1}^{k(n)}\left(\frac{a}{c}\right)^{r+l} r l \mathbf{E}\left|U_{n, l}\right|\left|U_{n, r}-G^{* r}(s)\right| \\
& \leqslant \frac{6(1-a / c)^{2}}{\varepsilon} \sum_{r=1}^{k(n)} \sum_{l=1}^{k(n)}\left(\frac{a}{c}\right)^{r+l} r l\left[\mathbf{E} U_{n, l}^{2}\right]^{1 / 2}\left[\mathbf{D} U_{n, r}\right]^{1 / 2} .
\end{aligned}
$$

Используя соотношения (48)-(51), получим оценки

$$
\mathbf{D} U_{n, r} \leqslant \frac{r s^{2 r}}{n a^{2 r}}, \quad \mathbf{E} U_{n, l}^{2}=\mathbf{D} U_{n, l}+\left(G^{* l}(s)\right)^{2} \leqslant \frac{l s^{2 l}}{n a^{2 l}}+1 .
$$


Подставляя эти оценки в (56) и используя неравенство $\sqrt{1+x} \leqslant 1+\sqrt{x}$, $x \geqslant 0$, убеждаемся, что условие (30) гарантирует стремление величины $B_{n 3}^{(2)}$ к нулю при $n \rightarrow \infty$. Теперь утверждение леммы следует из соотношений $(52),(44),(43)$ и (37). Лемма доказана.

3 а м е ч а н и е 1 . Условие (30) означает, что если $s<c$, то $k(n)$ может возрастать довольно быстро, например, $k(n)$ может равняться $n$. Если $s=c$, то $k(n)=o\left(n^{1 / 5}\right)$. Если же $s>c$, что типично, то $k(n)$ может расти весьма медленно, например, как $\alpha \ln n$ с $\alpha<1 /(\ln s-\ln c)$.

3 а м е ч а н и е 2 . Используя определение (33), несложно убедиться, что неравенство (50) можно уточнить следующим образом:

$$
0 \leqslant h_{k}\left(x_{1}, \ldots, x_{k}\right) \leqslant \min \left\{\frac{s^{k}}{a^{k}}, \frac{x_{1} \cdots x_{k}}{a^{k}}\right\} .
$$

Поэтому если предположить, что сушествует второй момент $\mu_{2}=\mathbf{E} X_{1}^{2}$, то основное неравенство (51) можно уточнить:

$$
\mathbf{D} U_{n, k} \leqslant \frac{k}{n} \mathbf{E}\left[\min \left\{\frac{s^{k}}{a^{k}}, \frac{X_{1} \cdots X_{k}}{a^{k}}\right\}\right]^{2} \leqslant \frac{k}{n} \min \left\{\frac{s^{2 k}}{a^{2 k}}, \frac{\mu_{2}^{k}}{a^{2 k}}\right\} .
$$

С учетом этого неравенства, условие (30) можно заменить более точным соотношением

$$
\frac{1}{\sqrt{n}} \sum_{r=1}^{k(n)}\left(\frac{\min \left\{s, \mu_{2}\right\}}{c}\right)^{r} r^{3 / 2} \max \left\{1, \sum_{l=1}^{k(n)}\left(\frac{s}{c}\right)^{l} l\right\} \rightarrow 0 \quad(n \rightarrow \infty) .
$$

Лемма 6. Пусть $\left\{\xi_{n}\right\}_{n \geqslant 1} u\left\{\eta_{n}\right\}_{n \geqslant 1}-$ последовательности случайных величин, причем $\xi_{n} \Longrightarrow \xi u \eta_{n} \stackrel{\mathbf{P}}{\longrightarrow} b$ nрu $n \rightarrow \infty$, где $b \in \mathbb{R}^{1}$, a $\xi-$ некоторая случайная величина. Тогда $\xi_{n} \eta_{n} \Longrightarrow b \xi(n \rightarrow \infty)$.

Д о к а з а т е л ь с т в о см. в [11], раздел 20.6 (с. 281-282).

Несмотря на то, что следуюшее утверждение имеет вспомогательный характер, в силу его важности мы оформим его в виде теоремы.

Теорема 2. Пусть оченка $\psi_{n}(s)$ определяется соотношениями (10) и (11), причем функиия $m(n) \leqslant n$ неограниченно возрастает при $n \rightarrow \infty$ так, что выполнено (17). Пусть оченка $\sigma_{n}^{2}$ определяется соотношениями (31)-(33), причем последовательность натуральных чисел $\{k(n)\}_{n \geqslant 1}$ удовлетворяет условиям $1 \leqslant k(n) \leqslant n, k(n) \rightarrow \infty$ $u(30)$. Тогда случайная величина $\sigma_{n}^{-1} \sqrt{n}\left(\psi_{n}(s)-\psi_{0}(s)\right)$ асимптотически нормальна:

$$
\mathbf{P}\left\{\sigma_{n}^{-1} \sqrt{n}\left(\psi_{n}(s)-\psi_{0}(s)\right)<x\right\} \Longrightarrow \Phi(x) \quad(n \rightarrow \infty) .
$$

Д о к а з а т ел ь с т в о. Положим

$$
\xi_{n}=\sigma^{-1} \sqrt{n}\left(\psi_{n}(s)-\psi_{0}(s)\right), \quad \eta_{n}=\sigma_{n}^{-1} \sigma .
$$

По лемме $3 \mathbf{P}\left\{\xi_{n}<x\right\} \Longrightarrow \Phi(x)$, а по лемме $5 \eta_{n} \stackrel{\mathbf{P}}{\longrightarrow} 1$ при $n \rightarrow \infty$. Теперь требуемое следует из леммы 6 . Теорема доказана. 
Теперь перейдем к построению оценки вероятности разорения для обобщенного процесса риска по наблюдениям за имеющейся траекторией процесса до некоторого момента времени $t$. Из приведенных выше рассуждений ясно, что в качестве оценки асимптотической дисперсии $\sigma^{2}$ следует взять оценку $\sigma_{N(t)}^{2}$, положив

$$
\sigma_{N(t)}^{2}=\left(1-\frac{a}{c}\right)^{2} \sum_{r=1}^{k(N(t))} \sum_{l=1}^{k(N(t))}\left(\frac{a}{c}\right)^{r+l} r l \bar{\sigma}_{r, l}
$$

где

$$
\begin{gathered}
\bar{\sigma}_{r, l}=\frac{1}{N(t)} \sum_{i=1}^{N(t)} \bar{h}_{r}\left(X_{i}\right) \bar{h}_{l}\left(X_{i}\right)-U_{N(t), r} U_{N(t), l}, \\
\bar{h}_{j}(x)=\frac{1}{a} \int_{0}^{\infty} U_{N(t), j-1}(s-y) \mathbf{1}_{[y, \infty)}(x) d y, \\
U_{N(t), k}=U_{N(t), k}(s)=\frac{1}{C_{N(t)}^{k}} \sum_{1 \leqslant i_{1}<\cdots<i_{k} \leqslant N(t)} h_{k}\left(X_{i_{1}}, \ldots, X_{i_{k}}\right), \\
h_{k}\left(x_{1}, \ldots, x_{k}\right) \\
=\frac{1}{a^{k}} \int_{0}^{\infty} \cdots \int_{0}^{\infty} \mathbf{1}_{[0, s)}\left(y_{1}+\cdots+y_{k}\right) \prod_{j=1}^{k} \mathbf{1}_{\left[y_{j}, \infty\right)}\left(x_{j}\right) d y_{1} \cdots d y_{k} .
\end{gathered}
$$

Чтобы сформулировать утверждение о состоятельности оценки $\sigma_{N(t)}^{2}$ при $t \rightarrow \infty$, нам понадобится еще одно вспомогательное утверждение.

Лемма 7. Пусть $\left\{\xi_{j}\right\}_{j \geqslant 1}$ - последовательность случайных величин, слабо сходящаяся $\kappa$ некоторой случайной величине $\xi$ при $j \rightarrow \infty$. Пусть $\left\{N_{n}\right\}_{n \geqslant 1}$ - последовательность челочисленньх положительных случайных величин такая, что при каждом $n \geqslant 1$ случайная величина $N_{n}$ независима от последовательности $\left\{\xi_{j}\right\}_{j \geqslant 1} u N_{n} \stackrel{\mathbf{P}}{\longrightarrow} \infty$ nри $n \rightarrow \infty$. Тогда $\xi_{N_{n}} \Longrightarrow \xi(n \rightarrow \infty)$.

Д о к а з а т е л ь с т в о этого результата можно найти, например, в [12] (см. теорему 5.1.1 там).

Теперь мы можем сформулировать следующее утверждение о состоятельности оценки $\sigma_{N(t)}^{2}$.

Лемма 8. Пусть оиенка $\sigma_{N(t)}^{2}$ определяется соотношениями (57)-(61), в которых последовательность натуральных иисел $\{k(n)\}_{n \geqslant 1}$ удовлетворяет условиям $1 \leqslant k(n) \leqslant n, k(n) \rightarrow \infty u(30)$. Предположим, что $\Lambda(t) \stackrel{\mathbf{P}}{\longrightarrow} \infty$ npu $t \rightarrow \infty$. Тогдa

$$
\sigma_{N(t)}^{-1} \sigma \stackrel{\mathbf{P}}{\longrightarrow} 1 \quad(t \rightarrow \infty) .
$$

Д ок а з а т е л ь с т в о. По лемме 1 из условия $\Lambda(t) \stackrel{\mathbf{P}}{\longrightarrow} \infty$ вытекает, что $N(t) \stackrel{\mathbf{P}}{\longrightarrow} \infty$. Теперь требуемое следует из лемм 5 и 7 . Лемма доказана. 
5. Интервальные оценки вероятности разорения для обобщенного процесса риска. Основную роль в данном пункте играет следуюшее утверждение.

Теорема 3. Пусть оченка $\psi_{N(t)}(s)$ определяется соотношениями (12) и (13), причем бункиия $m(n)$ такова, что $m(n) \rightarrow \infty n p u$ $n \rightarrow \infty$ так, что выполнено условие (17). Пусть оченка $\sigma_{N(t)}^{2}$ определяется соотношениями (57)-(61), в которьх последовательность натуральных чисел $\{k(n)\}_{n \geqslant 1}$ удовлетворяет условиям $1 \leqslant k(n) \leqslant n$, $k(n) \rightarrow \infty u(30)$. Предположим, ито $\Lambda(t) \stackrel{\mathbf{P}}{\longrightarrow} \infty$ nрu $t \rightarrow \infty$. Тогда

$$
\mathbf{P}\left\{\sigma_{N(t)}^{-1} \sqrt{N(t)}\left(\psi_{N(t)}(s)-\psi(s)\right)<x\right\} \Longrightarrow \Phi(x) \quad(t \rightarrow \infty) .
$$

Д о к а з а т е л ь с т в о. Данное утверждение является непосредственным следствием теоремы 2 и леммы 7.

Для $\delta \in(0,1) \delta$-квантиль стандартного нормального распределения будем обозначать $u_{\delta}$. При больших $t$ из (62) вытекает, что

$$
\mathbf{P}\left\{\sigma_{N(t)}^{-1} \sqrt{N(t)}\left(\psi_{N(t)}(s)-\psi(s)\right)<x\right\} \approx \Phi(x) .
$$

Поэтому для $\gamma \in(0,1)$ приближенный $100 \gamma$-процентный доверительный интервал для вероятности разорения $\psi(s)$ будет иметь вид

$$
\psi_{N(t)}(s)-\frac{u_{(\gamma+1) / 2} \sigma_{N(t)}}{\sqrt{N(t)}} \leqslant \psi(s) \leqslant \psi_{N(t)}(s)+\frac{u_{(\gamma+1) / 2} \sigma_{N(t)}}{\sqrt{N(t)}} .
$$

Наряду с теоремой 3, мы можем сформулировать еше один асимптотический результат.

Теорема 4. Пусть выполнены условия теоремы 3 и существуют неограниченно возрастающая функиия $d(t)$ и случайная величина $Y$ такие, ито имеет место сходимость (19). Тогда

$$
\mathbf{P}\left\{\sigma_{N(t)}^{-1} \sqrt{d(t)}\left(\psi_{N(t)}(s)-\psi(s)\right)<x\right\} \Longrightarrow \mathbf{E} \Phi(x \sqrt{Y}) \quad(t \rightarrow \infty)
$$

Д о к а з а т е л ь с т в о. По теореме 1 мы имеем

$$
\mathbf{P}\left\{\sigma^{-1} \sqrt{d(t)}\left(\psi_{N(t)}(s)-\psi(s)\right)<x\right\} \Rightarrow \mathbf{E} \Phi(x \sqrt{Y}) \quad(t \rightarrow \infty) .
$$

Положим

$$
\eta_{t}=\frac{\sigma_{N(t)}^{-1}}{\sigma^{-1}}, \quad \xi_{t}=\sigma^{-1} \sqrt{d(t)}\left(\psi_{N(t)}(s)-\psi(s)\right)
$$

Теперь требуемое вытекает из леммы 6 с учетом (66) и леммы 8. Теорема доказана.

Если $\delta$-квантиль функции распределения $\Psi(x)=\mathbf{E} \Phi(x \sqrt{Y})$ обозначить $w_{\delta}$, то при известном распределении случайной величины $Y$, наряду 
с (64), с помощью (65) для $\gamma \in(0,1)$ мы можем построить еще один приближенный $100 \gamma$-процентный доверительный интервал для вероятности разорения $\psi(s)$ :

$$
\psi_{N(t)}(s)-\frac{w_{(\gamma+1) / 2} \sigma_{N(t)}}{\sqrt{d(t)}} \leqslant \psi(s) \leqslant \psi_{N(t)}(s)+\frac{w_{(\gamma+1) / 2} \sigma_{N(t)}}{\sqrt{d(t)}}
$$

В частности, если $N(t)$ - стандартный пуассоновский процесс, что соответствует $\Lambda(t) \equiv t$, то естественно положить $d(t) \equiv t$. В этом случае, как легко видеть, $\mathbf{P}\{Y=1\}=1$, так что $\Psi(x) \equiv \Phi(x)$, и тем самым приближенный $100 \gamma$-процентный доверительный интервал (67) для вероятности разорения в случае классического процесса риска принимает вид

$$
\psi_{N(t)}(s)-\frac{u_{(\gamma+1) / 2} \sigma_{N(t)}}{\sqrt{t}} \leqslant \psi(s) \leqslant \psi_{N(t)}(s)+\frac{u_{(\gamma+1) / 2} \sigma_{N(t)}}{\sqrt{t}} .
$$

В заключение авторы хотели бы выразить искреннюю признательность рецензенту, внимательно прочитавшему статью и указавшему на некоторые недочеты, исправление которых, несомненно, способствовало улучшению изложения.

\section{СПИСОК ЛИТЕРАТУРЫ}

1. Бенинг В.Е., Королев В. Ю. Асимптотическое поведение обобщенных процессов риска. - Обозрение прикл. промышл. матем., сер. финансовая и страховая математика, 1998, т. 5, в. 1, с. 116-133.

2. Bülmann H. Tendencies of development in risk theory. - Centennial Celebration of the Actuarial Profession in North America. V. 2. Shaumburg: The Society of Actuaries, 1989, p. 499-521.

3. Эмбрехтс П., Клюппельберг K. Некоторые аспекты страховой математики. Теория вероятн. и ее примен., 1993, т. 38, в. 2, с. 374-416.

4. Beekman J. A. Collective risk results. - Trans. Soc. Actuaries, 1968, v. 20, p. 182.

5. Asmussen S. Applied Probability and Queues. Chichester: Wiley, 1987, $318 \mathrm{p}$.

6. Gnedenko B. V., Korolev V. Yu. Random Summation: Limit Theorems and Applications. Boca Raton, FL: CRC Press, 1996, 267 p.

7. Королев В.Ю. О сходимости распределений обобщенных процессов Кокса к устойчивым законам. - Теория вероятн. и ее примен., 1998, т. 43, в. 4, с. 786-792.

8. Croux K., Veraverbeke $N$. Nonparametric estimators for the probability of ruin. Insurance: Math. Econ., 1990, v. 9, № 2/3, p. 127-130.

9. Королев В. Ю. Сходимость случайных последовательностей с независимыми случайными индексами. II. - Теория вероятн. и ее примен., 1995, т. 40, в. 4, с. $907-$ 910.

10. Королюк B. С., Боровских Ю. В. Теория $U$-статистик. Киев: Наукова думка, $1989,383 \mathrm{c}$.

11. Крамер Г. Математические методы статистики. М.: Мир, 1975, 648 с.

12. Круглов В.М., Королев В.Ю. Предельные теоремы для случайных сумм. М.: Изд-во Моск. ун-та, 1990, 269 с.

13. Бенинг В.E., Королев В.Ю. Статистическое оценивание вероятности разорения для обобщенных процессов риска. - Теория вероятн. и ее примен., 1999, т. 44, B. 1 , c. 161-164. 